\title{
Tactile sensing and feedback in SEMG hand
}

\section{Leilei Zhang, Zujia Zheng and Gongfa Li ${ }^{\star}$}

Key Laboratory of Metallurgical Equipment and Control Technology, Ministry of Education,

Wuhan University of Science and Technology,

Wuhan, 430081, China

and

Biological Manipulators and Intelligent

Measurement of Control Research Center,

Wuhan University of Science and Technology,

Wuhan, 430081, China

Email: zhang.leilei1988@foxmail.com

Email: 302729582@qq.com

Email: ligongfa@wust.edu.cn

*Corresponding author

\section{Ying Sun, Guozhang Jiang and Jianyi Kong}

Equipment and Control Technology,

Ministry of Education,

Wuhan University of Science and Technology,

Wuhan, 430081, China

and

Hubei Key Laboratory of Mechanical Transmission and

Manufacturing Engineering,

Wuhan University of Science and Technology,

Wuhan, 430081, China

Email: sunying65@wust.edu.cn

Email:whjgz@wust.edu.cn

Email: 15697188659@wo.com.cn

\section{Bo Tao and Shuang Xu}

Key Laboratory of Metallurgical Equipment and Control Technology, Ministry of Education,

Wuhan University of Science and Technology,

Wuhan, 430081, China

and

Biological Manipulators and Intelligent

Measurement of Control Research Center,

Wuhan University of Science and Technology,

Wuhan, 430081, China

Email: botaoq@wust.edu.cn

Email: shuangxu@wust.edu.cn 


\title{
Hui Yu and Honghai Liu
}

Intelligent Systems and Biomedical Robotics Group,

School of Computing,

University of Portsmouth,

Portsmouth, PO1 3HE, UK

Email: hui.yu@port.ac.uk

Email: honghai.liu@port.ac.uk

\begin{abstract}
Active hand prostheses controlled using surface electromyography (SEMG) signals have been used for decades to restore the grasping function. Amputees with myoelectric hands wish to control the prostheses according to their own will and act like human hands as much as possible. Therefore, substantial research efforts have been put forth to advance the control of myoelectric hands. However, the tactile sensing and feedback of the myoelectric hands are still missing, thus limit hand grasp capabilities. In fact, integration of tactile sensing and feedback with hand prostheses plays an important role in improving the manipulation performance and enhancing perceptual embodiment for users. This paper reviews current state-of-the-art of tactile sensing technologies, including tactile sensor types and integration methods. Then, introduces the basic theory of SEMG signals and presents an overview of the sensory feedback employed to prosthetic hand. The paper concludes with a detailed discussion of challenging issues and future developments.
\end{abstract}

Keywords: tactile sensing; tactile feedback; SEMG signal; review; prosthetic hands.

Reference to this paper should be made as follows: Zhang, L., Zheng, Z., Li, G., Sun, Y., Jiang, G., Kong, J., Tao, B., Xu, S., Yu, H. and Liu, H. (xxxx) 'Tactile sensing and feedback in SEMG hand', Int. J. Computing Science and Mathematics, Vol. X, No. Y, pp.xxx-xxx.

Biographical notes: Leilei Zhang received his BS in Mechanical Design Manufacture and Automation major from the Shenyang Ligong University, Shenyang, China. He is currently occupied in his MS in Mechanical Engineering at the Wuhan University of Science and Technology. His current research interests include mechanical CAD/CAE, signal analysis and processing.

Zujia Zheng received his MS in the Wuhan University of Science and Technology, Wuhan, China. He is currently an Assistant Engineer in the Wuhan University of Science and Technology. His major research interest is application of intelligent robot technologies in civil engineering.

Gongfa $\mathrm{Li}$ received his $\mathrm{PhD}$ in the Wuhan University of Science and Technology, Wuhan, China. He is currently a Professor in the Wuhan University of Science and Technology. His major research interests are computer aided engineering, mechanical $\mathrm{CAD} / \mathrm{CAE}$, modelling and optimal control of complex industrial process.

Ying Sun is currently an Associate Professor in the Wuhan University of Science and Technology. Her major research focuses on teaching research in Mechanical Engineering. 
Guozhang Jiang received his $\mathrm{PhD}$ in the Wuhan University of Science and Technology, China. He is currently a Professor in the Wuhan University of Science and Technology. His research interests are computer aided engineering, mechanical $\mathrm{CAD} / \mathrm{CAE}$ and industrial engineering and management system.

Jianyi Kong received his PhD in the Helmut Schmidt Universitat, Germany. He is currently a Professor in the Wuhan University of Science and Technology. His research interests are intelligent machine and controlled mechanism, mechanical and dynamic design and fault diagnosis of electrical system, mechanical CAD/CAE, intelligent design and control.

Bo Tao received the PhD in University of Science and Technology of China, China. He is currently a Lecturer in Wuhan University of Science and Technology. His research interests are artificial intelligence and sensing technology.

Shuang $\mathrm{Xu}$ received the $\mathrm{PhD}$ in Huazhong University of Science and Technology, China. She is currently a Lecturer in Wuhan University of Science and Technology. Her research interests are artificial intelligence and image processing.

Hui $\mathrm{Yu}$ received his $\mathrm{PhD}$ in the Brunel University, London .He is currently the Leader of Computer and Creative Technology in the University of Portsmouth. His research interests are human face analysis, human motion analysis, 3D modelling technology and human-computer interaction.

Honghai Liu received his $\mathrm{PhD}$ in Intelligent Robotics from the Kings College London, London, UK. He is currently a Chair Professor of intelligent systems and robotics. His research interests include wearable sensing, biomechatronics, intelligent video analytics, intelligent robotics and their applications with an emphasis on approaches that could make contribution to the intelligent connection of perception to action using contextual information.

\section{Introduction}

The 'sense of touch' can be defined as the information acquired by human tactile perception system through the contact or operation of objects. It is an important sensory function when people contact with the external environment (Kappassov et al., 2015). Tactile information is an important medium for communicating with the environment, whether for human beings or robots (Yousef et al., 2011). The sense of touch itself is very sensitive and it can directly measure the various properties of objects and environments. Humans use the tactile organs of the skin to sense the temperature of the object, grasp objects reasonably and identify objects with the same in visual but different in materials by touch. For the prosthetic hand, tactile information can provide the contact force between the fingers and the objects, the contact position and other important information, which can improve fine grasping ability of the dexterous hand. It is of great importance for prostheses users to explore and operate unknown objects in the external environment. 
The main task of tactile sensing is to obtain the object and environment information and to detect or perceive a series of physical characteristics when the prosthetic hand interacts with the object and environment (Tegin and Wikander, 2013). It not only can obtain the contact position of the finger and the object, as well as the contact force distribution function, but also can obtain the information which the vision sensor cannot obtain, as an example the mechanical properties, thermal character and vibration performance of the object.

But for an ideal prosthetic hand (Miao et al., 2015; Ulrika and Ingela, 2015; Peerdeman et al., 2011), it is not enough to have tactile sensing only. Regarding hand capability, an ideal prosthetic hand should have tactile sensation to explore surrounding objects in human-centred environments and deliver tactile feedback in a natural manner. The control of grasping largely depends on tactile feedback and providing feedback to the user would help to operate a prosthetic hand more efficiently. Tactile feedback can deliver the information such as shapes of the object, material property, work position and contact state (friction, sliding) to the human nervous system, so as to realise a closed-loop interaction between human and prosthetic hand (Li et al., 2017). Therefore, a common thought is that prostheses would function better if integrate tactile sensing and feedback with prosthetic hand and make use of tactile information acquired by sensors.

\section{Tactile sensing technologies}

Tactile sensors can be defined as a tool that can evaluate a given property of an object through physical contact between the hand and the object (Dang and Allen, 2014). Sensors are made of sensitive material or structure and they are mainly used to measure the change of physical properties caused by the interaction between themselves and the external object. Change of capacitance, resistance, optical distribution, electrical charge can be used in the sensing systems. For the prosthetic hand, grasping is one of the basic skill expected to have, most research on tactile sensing focuses on the control of grasp force and pressure to realise stable grasp and fine manipulation (Dang and Allen, 2014). The measured characteristics of touch, however, can be not only force and pressure, but also object parameters such as stiffness, texture and shape (Fishel and Loeb, 2012). Therefore, different transduction techniques are needed to realise a humanoid tactile sensing system.

\subsection{Tactile sensor integration techniques}

Compared with the dexterous robot hand, the shape, size and weight of the prosthetic hand have more stringent requirements and it also needs to meet the technical requirements such as speed and grasping force (Jiang et al., 2017). Therefore, it is a challenging problem to realise the integration of the structure, actuation, sensing and control of the prosthetic limb under the constraint of size, shape and weight.

When the prosthetic hand performs the task of grasping or manipulation, the tactile sensors provide the contact force, position and sliding information. The integrated design of tactile sensor array consists of two problems (Jiang et al., 2017; Liu et al., 2012): 
a Simplified design and unit layout for tactile sensor array. According to the prosthetic operation function and the demand of perceptual feedback, the configuration of the tactile sensor array is optimised. Tactile sensors should be either flexible shaped to envelop a given surface and rigid as an attachment part.

b Intensive design of information acquisition system for tactile sensor array. If each sensitive unit is connected to the printed circuit boards (PCB) of the sensing array, the amount of wires required to read and transmit the data from the sensing arrays will increase.

An effective way of integrating tactile sensors is to embed them into the robot hand. The embedding procedure of the tactile sensing skin within the robot hand involves the following steps (Schmitz et al., 2011):

- Definition of the surface to be covered by the available computer-aided-drawing (CAD) model or by means of a 3D scanner.

- Manufacturing of the supporting part for the sensor using tactile sensing PCB. This part is to be attached to the robot hand. The use of a 3D printer can facilitate the manufacturing procedure.

- Identification and wiring of the sensing elements.

- Gluing the sensing elements down on the supporting part.

- Covering the sensing elements with flexible material, e.g., silicon rubber. For a specific surface shape, custom moulds should be designed.

\subsection{Properties of the tactile sensor used in prosthetic hand}

A tactile sensor that meets the requirements of prosthetic hand operation should have the following characteristics: high spatial resolution, high sensitivity, high frequency response, low hysteresis, simple wiring and large surface friction coefficient. High spatial resolution can detect more mechanical information, which can greatly improve the recognition ability of the object shape and the grasping ability of prosthetic hand. However, the increase in resolution can cause three problems: the complex of wire arrangement, the increase of information processing and the reduction of signal to noise ratio. High sensitivity is beneficial to detect low contact force and improve the ability of prosthetic hand to grasp fragile objects, but somehow, it will reduce the detection range of sensor. Frequency response can detect the change of pressure more quickly and improve the real-time performance of prosthetic hand, which is the key performance of slip detection. But yet, the high frequency response requires the high hardness of the cover material of the sensitive element, which will have a negative impact on the grasp stability of prosthetic hand. Because of the above reasons, the prosthetic hand commonly used elastic material as a protective layer of the sensor. When the pressure is loaded and released, the deformation and recovery of the elastic layer will delay, which will lead to hysteresis effect and reduce the spatial resolution and sensitivity of the sensor (Balasubramanian and Santos, 2014). 


\section{SEMG signal techniques}

\subsection{SEMG signals}

With the development of multi sensor technology, electronic information technology, pattern recognition technology and biomedical engineering, study on surface electromyography (SEMG) controlled prosthetic hand has gained growing attention (Sun et al., 2018). The prosthetic hand based on SEMG signals has become a hot topic in the field of prosthetic control.

The electromyographic (EMG) signal is the summation of the action potentials discharged by the active muscle fibres in the proximity of the recording electrodes (Jiang et al., 2010). As described by Farina and Holobar (2015), the EMG signal can be seen as a neural recording from a peripheral muscle that biologically amplifies neural signals of tens to hundreds motor neuron. A single motor neuron and its corresponding muscle fibres constitute a muscle unit (MU). The SEMG obtains EMG signals by placing electrodes on the skin. It is a kind of bioelectric signal recorded and included by electrodes when neuromuscular system is active. The activity of motor neurons activates the generation of muscle fibre action potentials and a compound action potential recorded at the skin surface is the SEMG recording.

\subsection{SEMG feature extraction and analysis}

When the SEMG signals are recorded from the muscles, they will be processed and analysed for activating certain prosthetic functions of the prosthesis. SEMG acquisition and processing are shown in Figure 1.

Figure 1 The frame diagram of SEMG acquisition and processing

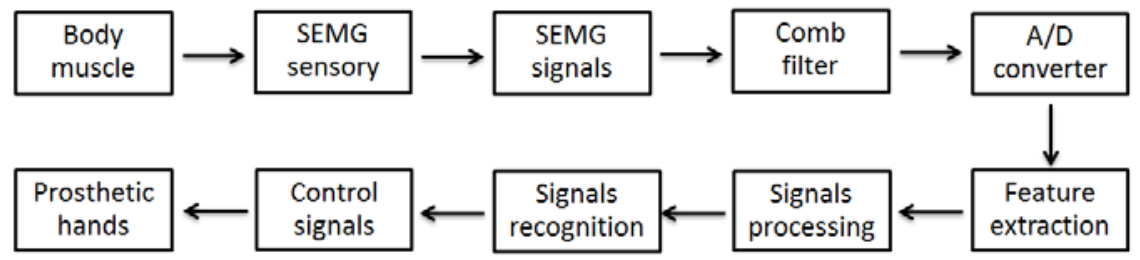

The purpose of signal analysis and processing is to discuss the possible cause of SEMG signals change and reflect activity and function of muscles effectively by the change of SEMG signals. The task of signal recognition is to translate the input SEMG signals into corresponding motion control commands. The feature extraction of SEMG signal is considered as one of the most important steps in pattern recognition. The classification of feature quantity will directly determine the classification effect of the algorithm ( $\mathrm{He}$ et al., 2017). To improve the performance of the classifier, researchers have been using different types of SEMG features as an input to the classifier. To achieve optimal classification performance, the properties of SEMG feature space (e.g., maximum class separability, robustness and the computational complexity) should be taken into consideration (Fang et al., 2015). 


\section{Tactile feedback}

Tactile feedback is a method to provide sensory information to the body, through a sensory channel different from that normally used (e.g., substitute touch with hearing) or through the same channel but in a different modality (e.g., substitute pressure with vibration) (Kaczmarek et al., 1991). The success of the approach depends on the user's ability to interpret the type and location of the stimulus and associate it with the prosthesis. The most common method is to translate tactile information from the prosthesis to the amputee using vibration, electrotactile or auditory substitution.

\subsection{Vibrotactile feedback}

Vibrotactile feedback involves communicating sensory information from the prosthesis to the user through the application of mechanical vibration to the user's skin at forearm (Cipriani et al., 2012). The main features of the stimulus are vibration frequency, amplitude and duration of vibration, typically at frequencies in the range of $10-500 \mathrm{~Hz}$ (Kaczmarek et al., 1991) and they can be modulated to convey different kinds of information like grasping forces and pressures present in the prosthesis.

The vibrotactile feedback in prosthetic has been widely researched due to its higher compatibility with EMG control and acceptability compared to electrotactile stimulation (Kaczmarek et al., 1991). And then, it has been widely researched due to its higher compatibility with EMG control and acceptability compared to electrotactile stimulation (Kaczmarek et al., 1991). Explore on vibrotactile sensory substitution has been mostly applied to communicate tactile information during grasping tasks. Vibrotactile feedback systems have been used in research with the Otto Bock, motion control and iLimb myoelectric prostheses (Saunders and Vijayakumar, 2011; Chatterjee et al., 2008; Sears et al., 2008). Recent studies (Cipriani et al., 2012; Tejeiro et al., 2012) have reported that vibratory feedback was shown to improve user performance through a better control of grip force and success rates in performing grasping tasks. Currently, a wearable vibrotactile haptic device has been developed for stiffness perception during an interaction with virtual objects, as shown in Figure 2(a). A vibrotactile stimulation system applied with a myoelectric prosthetic hand is illustrated in Figure 2(b). With the development of technologies, they are potential to be applied in sensory rehabilitation.

\subsection{Electrotactile feedback}

Electrotactile feedback is induced by a current that flows through the skin. Recently it was exploited in the Yokoi Hand to deliver grip force feedback on a single skin-site and by Geng et al. (2011) to investigate the effects of different patterns on the perception threshold. Electrotactile feedback system is comprised of force sensors that are placed on the fingers and palm of a prosthetic hand, interface circuits for processing the sensor data and electrodes that are placed on nearby skin (Cloutier and Yang, 2013). It communicates sensory information to the prosthetic user via electrodes placed on the user's skin. Electrotactile feedback can be used to elicit pressure and lip feedback. Sensory communication is most often achieved through modulation of the electrical current parameters: amplitude, frequency and pulse rate to single or multiple electrode sites (Antfolk et al., 2013). Through the experimental method, the relationship between 
electrical stimulation parameters and the grasping force in prosthesis should be determined to make user feel comfortable and safe and have a clear sense of excitement.

Figure 2 (a) Wearable haptic device with five vibrotactile actuators (b) vibrotactile feedback system applied with a myoelectric prosthetic hand) (see online version for colours)

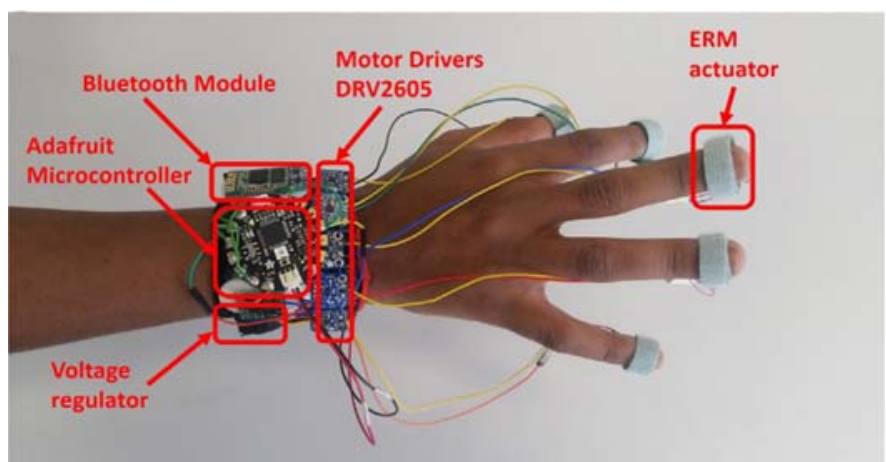

(a)

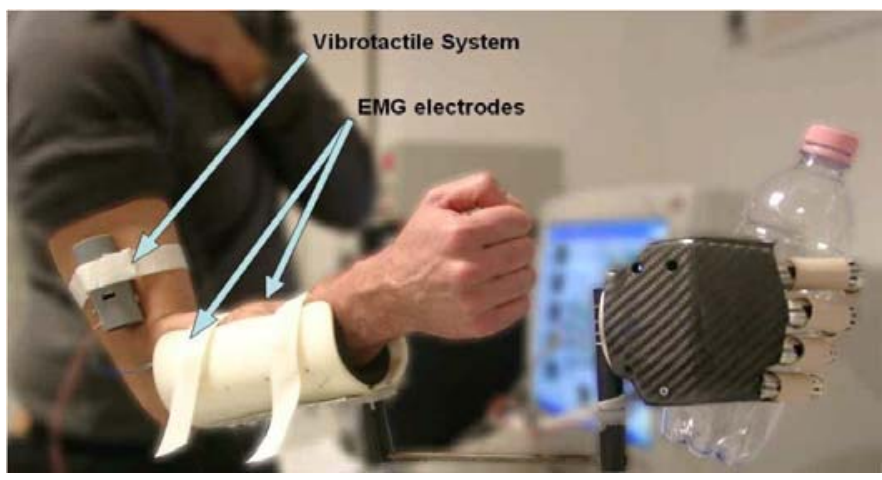

(b)

Source: ${ }^{\mathrm{a}}$ Maereg et al. (2017) and ${ }^{\mathrm{b}}$ Cipriani et al. (2008)

Figure 3 (a) Representing the distribution of force vectors on a fingertip using the electrotactile display (b) electrodes of electrotactile display (see online version for colours)

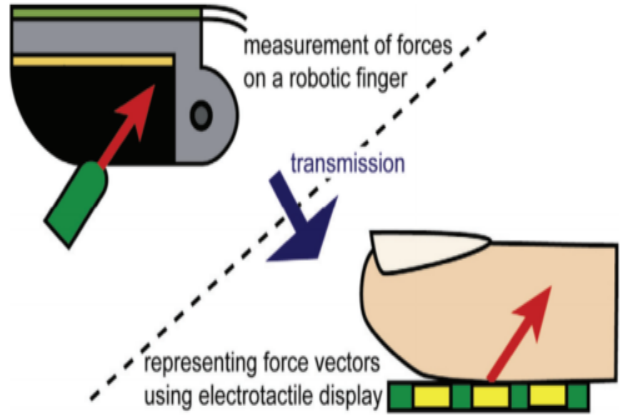

(a)

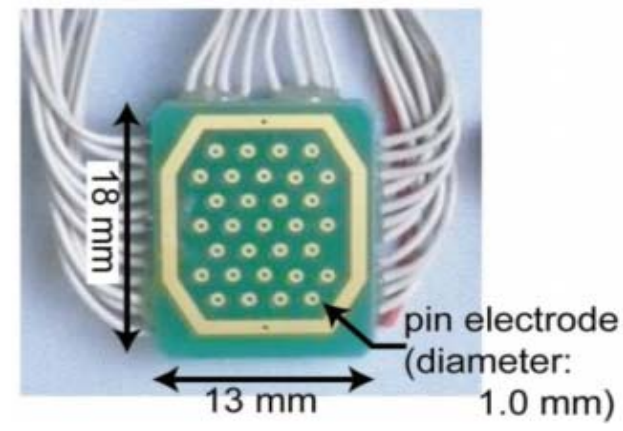

(b)

Source: Sato and Tachi (2010) 
Sato and Tachi (2010) proposed a design of electrotactile stimulation for distribution of force vectors that can be represented by an electrotactile display; the principle is shown in Figure 3(a). The structure of an electrotactile display is shown in Figure 3(b).

\subsection{Auditory feedback}

Auditory feedback has been demonstrated as a technique to convey contact of a robotic hand to an object as well as the position of the hand's digits and intended grasping pattern (Gonzalez et al., 2011, 2012a). Methods of auditory feedback provide information on the state of a robotic or prosthetic hand through varying frequencies of tones or sounds.

Gonzalez et al. (2012b) designed an experience to explore the effect of using an auditory display as a sensory feedback system for reaching and grasping movements for prosthetic applications. The results showed that the usage of an auditory display to monitor and control a robot hand improved the temporal and grasping performance greatly, while reducing mental effort and improving their confidence.

Gibson and Artemiadis (2015) presented a method of sensing tactile information in dexterous manipulation by multi-frequency auditory signals. By grasping several objects of varying stiffness and weight with EMG prosthetic hand, the tactical information was provided in time through the proposed auditory feedback. Results showed that users were able to adapt and learn the feedback technology after short use and could eventually use auditory information alone to control the grasping forces of a prosthetic hand.

\section{Conclusions}

Although some tactile sensing and feedback techniques have been developed to solve the problem of integration of tactile sensing and feedback with hand prostheses, but with limited success in commercial application. There are still much challenges and opportunities in the field of tactile sensation restoration.

For the most current tactile sensors, the tactile sensor arrays cannot fully meet the requirements of the prosthesis. But the multi sensor information fusion can not only enhance the detection accuracy of the fingertip contact force of prosthetic hand, but also improve the rolling contact accuracy of unknown objects. Therefore, multi sensor information fusion technology is expected to improve the manipulation performance of prosthetic hand.

In relation to SEMG signals, the processes of SEMG signal acquisition and feature extraction are more susceptible to external noise. Among the aforementioned tactile feedback, electrotactile feedback is believed to be the most promising technique its small dimension, low noise and friendly interface with electronics. However, electrical feedback signals and SEMG signals inevitably interfere with each other, especially the electrical feedback signals have a greater influence on the SEMG signals, which can lead to the degradation of the EMG signal quality. So how to restrain the interference between electrotactile feedback and SEMG signal is a key problem to be solved.

With regard to tactile stimulation feedback, challenges are natural sensation generation and multi-sensation restoration. The current feedback modalities can only provide one or two sensing information, such as force or pressure. But other tactile features, such as texture, shape and stiffness also need to be provided by feedback 
system. So, multiple tactile sensations are expected, furthermore, to be fed back simultaneously just like the way that human skin works. Therefore, it is expected that an effective sensory feedback which can generate natural tactile perception will facilitate clinical use in prosthetic hands or other application in virtual reality.

\section{Acknowledgements}

This work was supported by grants of the National Natural Science Foundation of China (Grant No. 51575407, 51575338, 51575412, 61273106) and the Grants of National Defense Pre-Research Foundation of the Wuhan University of Science and Technology (GF201705).

\section{References}

Antfolk, C., D'Alonzo, M., Controzzi, M. et al. (2013) 'Artificial redirection of sensation from prosthetic fingers to the phantom hand map on transradial amputees: vibrotactile versus mechanotactile sensory feedback', IEEE Transactions on Neural Systems and Rehabilitation Engineering, Vol. 21, No. 1, pp.112-120.

Balasubramanian, R. and Santos, V.J. (2014) The Human Hand as an Inspiration for Robot Hand Development, Springer Tracts in Advanced Robotics 95, Springer International Publishing, Switzerland.

Chatterjee, A., Chaubey, P., Martin, J. et al. (2008) 'Testing a prosthetic haptic feedback simulator with an interactive force matching task', Journal of Prosthetics and Orthotics, Vol. 20, No. 2, pp.27-34.

Cipriani, C., D'Alonzo, M. and Carrozza, M.C. (2012) 'A miniature vibrotactile sensory substitution device for multifingered hand prosthetics', IEEE Transactions on Biomedical Engineering, Vol. 59, No. 2, pp.400-408.

Cipriani, C., Zaccone, F., Micera, S. et al. (2008) 'On the shared control of an EMG-controlled prosthetic hand: analysis of user-prosthesis interaction', IEEE Transactions on Robotics, Vol. 24, No. 1, pp.170-184.

Cloutier, A. and Yang, J. (2013) 'Design, control, and sensory feedback of externally powered hand prostheses: a literature review', Critical Reviews in Biomedical Engineering, Vol. 41, No. 2 , p.161.

Dang, H. and Allen, P.K. (2014) 'Stable grasping under pose uncertainty using tactile feedback', Autonomous Robots, Vol. 36, No. 4, pp.309-330.

Fang, Y., Liu, H., Li, G. et al. (2015) 'A multichannel surface EMG system for hand motion recognition', International Journal of Humanoid Robotics, Vol. 12, No. 2, 1550011, 13pp, DOI: $10.1142 / \mathrm{S} 0219843615500115$.

Farina, D. and Holobar, A. (2015) 'Human-machine interfacing by decoding the surface electromyogram [life sciences]', IEEE Signal Processing Magazine, Vol. 32, No. 1, pp. $115-120$.

Fishel, J.A. and Loeb, G.E. (2012) 'Bayesian exploration for intelligent identification of textures', Frontiers in Neurorobotics, Vol. 6, No. 6, p.4.

Geng, B., Yoshida, K. and Jensen, W. (2011) 'Impacts of selected stimulation patterns on the perception threshold in electrocutaneous stimulation', Journal of Neuroengineering and Rehabilitation, Vol. 8, No. 1, pp.1-10.

Gibson, A. and Artemiadis, P. (2015) 'Neural closed-loop control of a hand prosthesis using cross-modal haptic feedback', IEEE International Conference on Rehabilitation Robotics, pp.37-42. 
Gonzalez, J., Soma, H., Sekine, M. et al. (2011) 'Auditory display as a prosthetic hand biofeedback', Journal of Medical Imaging and Health Informatics, Vol. 1, No. 4, pp.325-333.

Gonzalez, J., Soma, H., Sekine, M. et al. (2012a) 'Psycho-physiological assessment of a prosthetic hand sensory feedback system based on an auditory display: a preliminary study', Journal of Neuroengineering and Rehabilitation, Vol. 9, No. 1, p.33.

Gonzalez, J., Suzuki, H., Natsumi, N. et al. (2012b) 'Auditory display as a prosthetic hand sensory feedback for reaching and grasping tasks', Engineering in Medicine and Biology Society, p.1789.

He, Y., Li, G., Liao, Y., Sun, Y. et al. (2017) 'Gesture recognition based on an improved local sparse representation classification algorithm', Cluster Computing, No. 1, pp.1-12, DOI: $10.1007 / \mathrm{s} 10586-017-1237-1$.

Jiang, L., Yang, B., Huang, Q. et al. (2017) 'Biomechatronic integration of intelligent prosthetic hand', Robot, Vol. 39, No. 4, pp.387-394.

Jiang, N., Falla, D., D’Avella, A. et al. (2010) 'Myoelectric control in neurorehabilitation', Critical Reviews in Biomedical Engineering, Vol. 38, No. 4, p.381.

Kaczmarek, K.A., Webster, J.G., Bach-Y-Rita, P. et al. (1991) 'Electrotactile and vibrotactile displays for sensory substitution systems', IEEE Transactions on Biomedical Engineering, Vol. 38, No. 1, pp.1-16.

Kappassov, Z., Corrales, J.A. and Perdereau, V. (2015) Tactile sensing in dexterous robot hands - review', Robotics and Autonomous Systems, Vol. 74, Part A, pp.195-220, North-Holland Publishing Co..

Li, B., Sun, Y., Li, G. et al. (2017) 'Gesture recognition based on modified adaptive orthogonal matching pursuit algorithm', Cluster Computing, No. 3, pp.1-10, DOI: 10.1007/s10586-0171231-7.

Liu, H., Greco, J., Song, X. et al. (2012) 'Tactile image based contact shape recognition using neural network', IEEE Conference on Multisensor Fusion and Integration for Intelligent Systems, pp.138-143.

Maereg, A.T., Nagar, A.K., Reid, D. et al. (2017) 'Wearable vibrotactile haptic device for stiffness discrimination during virtual interactions', Frontiers in Robotics and AI, Vol. 4, DOI: $10.3389 /$ frobt.2017.0004.

Miao, W., Li, G., Jiang, G. et al. (2015) 'Optimal grasp planning of multi-fingered robotic hands: a review', Applied and Computational Mathematics, Vol. 14, No. 3, pp.238-247.

Peerdeman, B., Boere, D. and Witteveen, H. (2011) 'Myoelectric forearm prostheses: state of the art from a user-centered perspective', Journal of Rehabilitation Research and Development, Vol. 48, No. 6, pp.719-737.

Sato, K. and Tachi, S. (2010) 'Design of electrotactile stimulation to represent distribution of force vectors', Haptics Symposium, pp.121-128, DOI: 10.1109/HAPTIC.2010.5444666.

Saunders, I. and Vijayakumar, S. (2011) 'The role of feed-forward and feedback processes for closed-loop prosthesis control', Journal of Neuroengineering \& Rehabilitation, Vol. 8, No. 1, pp.1-12.

Schmitz, A., Maiolino, P., Maggiali, M. et al. (2011) 'Methods and technologies for the implementation of large-scale robot tactile sensors', IEEE Transactions on Robotics, Vol. 27, No. 3, pp.389-400.

Sears, H., Iversen, E. et al. (2008) 'Grip force feedback in an electric hand-preliminary results', Proceedings of the MEC'08 Conference.

Sun, Y., Li, C., Li, G. et al. (2018) 'Gesture recognition based on kinect and SEMG signal fusion', Mobile Networks and Applications [online] https://doi.org/10.1007/s11036-018-1008-0.

Tegin, J. and Wikander, J. (2013) 'Tactile sensing in intelligent robotic manipulation - a review', Industrial Robot, Vol. 32, No. 1, pp.64-70. 
Tejeiro, C., Stepp, C.E., Malhotra, M. et al. (2012) 'Comparison of remote pressure and vibrotactile feedback for prosthetic hand control', IEEE RAS \& EMBS International Conference on Biomedical Robotics and Biomechatronics, pp.521-525.

Ulrika, W. and Ingela, C. (2015) 'Forearm amputees' views of prosthesis use and sensory feedback', Journal of Hand Therapy Official Journal of the American Society of Hand Therapists, Vol. 28, No. 3, p.269.

Yousef, H., Boukallel, M. and Althoefer, K. (2011) 'Tactile sensing for dexterous in-hand manipulation in robotics-a review', Sensors and Actuators A: Physical, Vol. 167, No. 2, pp.171-187. 